\title{
Energy Efficient MAC protocols for Mobile Sensor Networks
}

\author{
Isha Batra \\ Master's in Engineering, \\ PEC, sec-12 \\ Chandigarh
}

\author{
Ritu Malik \\ Master's in Engineering \\ PEC, sec-12 \\ Chandigarh
}

\author{
Meenakshi Mittal \\ Master's in Engineering \\ PEC, sec-12 \\ Chandigarh
}

\begin{abstract}
A Mobile Sensor Network is a geographically distributed network which owes its name to the presence of mobile sink or sensor nodes within the network. In this paper we will present and discuss the energy-efficient contention-based and scheduled based medium access control (MAC) protocols for mobile sensor networks (MSNs). Most of the MAC protocols such as T-MAC, D-MAC, and the more commonly utilized SMAC proposed for wireless sensor networks assume sensors to be stationary after deployment, which usually provide very bad network performance in scenarios involving mobile sensors.

Handling mobility in wireless sensor networks in an energy-efficient way is a new challenge. We present new MAC layer approaches to support mobility in WSNs such as MS-MAC extends S-MAC to include mobility-awareness by decreasing this sleep duration when mobility is detected. S-MAC with Extended Kalman filter (EKF) reduces mobility-incurred losses by predicting the optimal data frame size for each transmission. M-LMAC (Mobile LMAC) as a TDMA-based MAC protocol which can support continuous mobility in the wireless sensor networks. MMAC utilizes a dynamic mobility-adaptive frame time to enhance TRAMA, a scheduled-based protocol, with mobility prediction. Likewise, G-MAC utilizes TDMA for cluster-based WSNs by combining the advantages of contention and contention-free MACs. All of the above MAC protocols are reviewed in detail.
\end{abstract}

The full text of the article is not available in the cache. Kindly refer the IJCA digital library at www.ijcaonline.org for the complete article. In case, you face problems while downloading the full-text, please send a mail to editor at editor@ijcaonline.org 